\title{
Mapping Protein Dynamics During Exocytosis of Single Microvesicles in Neuroendocrine Cells with Evanescent Field Mcroscopy
}

\author{
Justin W. Taraska $^{1 *}$, Kem A. Sochacki ${ }^{1}$, Agila Somasundaram ${ }^{1}$ \\ ${ }^{1}$ National Heart Lung and Blood Institute, National Institutes of Health, Bethesda, MD USA \\ *Corresponding author: justin.taraska@nih.gov
}

Cells of the body communicate with one another by releasing peptides, neurotransmitters, and small molecules by exocytosis. Triggered exocytosis in neurons and endocrine cells is a highly regulated process. Cells go to great lengths to ensure that exocytosis occurs at precisely the right time and location and that the correct amount of cargo is released during each exocytic burst. Once membrane-bound cargo is lost from the vesicle and enters the plasma membrane, it must be corralled and internalized by endocytosis. While many of the individual proteins important for this cycle have been identified, how these components associate with the vesicle assemble to drive fusion and regulate the recapture of material by endocytosis is not well understood.

To study exocytosis we imaged the fusion dynamics of a single microvesicles labeled with the vesicular acetylcholine transporter (VaChT) tagged to pHluorin, a pH-sensitive green fluorescent protein in living neuroendocrine cells with total internal reflection fluorescence microscopy (TIRF)[1]. TIRF selectively illuminates the cell at the coverslip/water interface with a sub-wavelength field of excitation light. Fluorescent material within this field is excited while fluorophores deeper into the cell are left dark. Thus, TIRF microscopy produces a wide-field high contrast image of events that occur within $\sim 100 \mathrm{~nm}$ of the bottom of the cell. With this method we show that during exocytosis VaChT rapidly diffuses out of single vesicles and into the plasma membrane. VaChT is next corralled over a dense network of membraneresident clathrin structures and packaged for endocytosis [1].

To further investigate factors that regulate microvesicle exocytosis we use dual-color TIRF imaging to observe membrane fusion with VAChT-pHluorin and a set of target proteins tagged with a red-fluorescent protein (mCherry, RFP)(Figure 1)[2]. These experiments show that many classic exocytic factors, including the SNAREs syntaxin1 and VAMP2, the SNARE modulator tomosyn, and Rab proteins, are pre-clustered at microvesicles and rapidly lost from the fusion site soon after exocytosis. Surprisingly, the ATPase NSF was transiently and locally recruited to vesicles at fusion. Furthermore, the endocytic BAR domain-containing proteins amphiphysin1, syndapin2, and endophilin were locally recruited during fusion. We additionally show that BAR proteins slow the loss of VAChT from fusion sites. A similar effect was seen with the over-expression of dynamins. These data suggest that proteins involved in classic clathrin-mediated endocytosis can also regulate microvesicle exocytosis. Our findings provide insights into the dynamics, assembly, and mechanistic roles of many key proteins in exocytosis and endocytosis at single sites of fusion in living cells.

These studies further illustrate the ability of TIRF microscopy, along with quantitative image analysis, to map the underlying structure of a complex cellular system and screen for new associations, patterns, and dynamics [3]. Investigating individual functional units of interacting molecules in their subcellular context will deepen our understanding of the self-organization, spatial patterns, dynamics, and control of biological systems. 
A VAChT-pHluorin

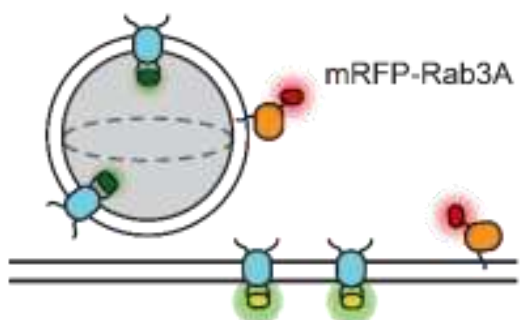

B
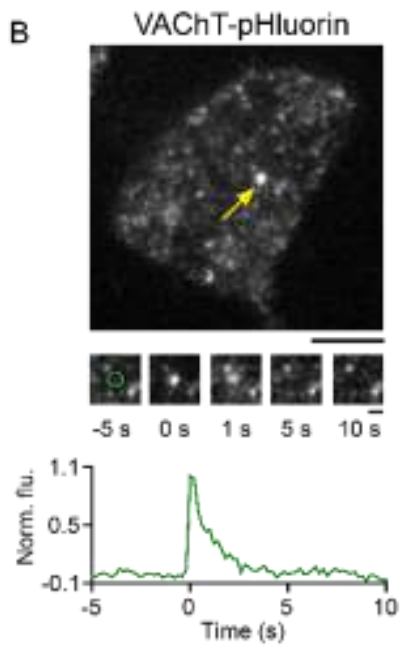

D
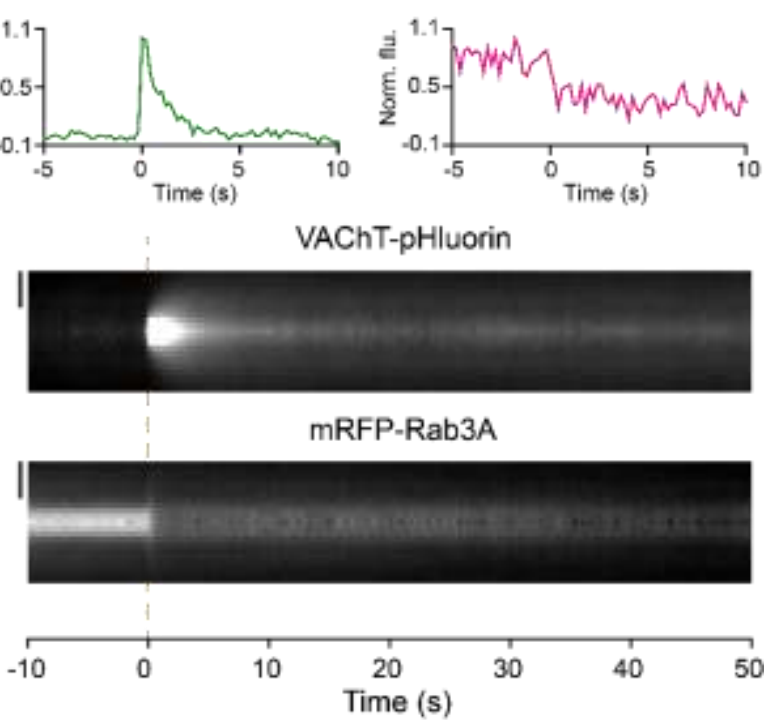

Figure 1. TIRF microscopy of protein dynamics at single fusion sites in live cells. (A) Cartoon of fluorescent protein fusions used to mark synaptic like microvesicles (VAChT-pHluorin and mRFP-Rab3a). During exocytosis VAChT-Phluorin brightens due to a pH change. B) TIRF images of a PC12 cell expressing VAChT-pHluorin (B) and mRFP-Rab3A (C). Arrows (yellow) show a fusion event in the green channel and the corresponding region in the red channel. Bar, $5 \mu \mathrm{m}$. (Middle) sub-regions containing a fusion event. Bar, 1 $\mu \mathrm{m}$ (Bottom) Plots of fluorescence intensities for the event shown above in both channels. (D) Average normalized radial line scan kymograph of VAChTpHluorin and mRFP-Rab3A during fusion from many events showing the dynamic spatial changes in fluorescence at single sites of exocytosis. Exocytosis is marked by orange line. Bar, $1 \mu \mathrm{m}$

\section{References:}

[1] Sochacki KA et al., Nature Communications. 3 (2012), p. 1154.

[2] Somasundaram A, Taraska JW, Mol. Biol. Cell. 29(2018),p.1891-1903.

[3] Larson BT et al., Mol. Biol. Cell. 25(2014), p.208493

[4] The authors acknowledge funding from the Intramural Research Program of the National Heart Lung and Blood Institute, National Institutes of Health, USA. 